\title{
INTERVENCIÓN EN UN CASO DE ENCOPRESIS SECUNDARIA EN EL CONTEXTO EDUCATIVO
}

\author{
JULIANA LÓPEZ DE LA LLAVE ${ }^{1}$, CARMEN PÉREZ-LLANTADA², ANDRÉS LÓPEZ \\ DE LA LLAVE ${ }^{2}$ y LOURDES LÓPEZ DE LA LLAVE ${ }^{2}$ \\ ${ }^{1}$ Ministerio de Educación y Cultura (MEC)' \\ ${ }^{2}$ Universidad Nacional de Educación a Distancia (UNED)
}

(Recibido el 10 de enero de 1999)

\begin{abstract}
Presentamos el caso de una niña de siete años tratada de encopresis secundaria mediante práctica positiva, moldeado, sobrecorrección y refuerzo positivo, minimizando el uso del castigo. Los episodios de encopresis quedaron reducidos a cero al quinto mes de la intervención, consiguiéndose, también, mejorar su aseo personal, aumentar el número de interacciones positivas con sus compañeros y reducir la emisión de conductas inadecuadas (levantarse, agredir, interrumpir, tirar objetos), todo ello en el contexto escolar al que se circunscribe la intervención. No obstante, se ha observado una notable interferencia entre la intervención y la consecución de los objetivos específicamente educativos.
\end{abstract}

Palabras clave: Encopresis secundaria, tratamiento conductual.

\section{Intervention of a secondary encopresis case in the school context}

A girl of seven years old was treated of secondary encopresis through positive practice and positive reinforcement, minimizing the use of the punishment. The encopresis episodes remained reduced to zero to the fifth month of the intervention. Also in the school context, was improved her personal bathroom, was increased the number of positive interactions with their companions and was reduced the improper conduct emission (be lifted, assaulted, interrupted, pulled objects). Nevertheless, it has been observed a notable interference between the intervention and the achievement of the educational goals in particular.

Key words: Secondary encopresis, behavioral treatment.

\section{INTRODUCCIÓN}

Entre las diversas definiciones de encopresis, las propuestas por Doleys (1978) y Levine (1975) son algunas de las más citadas en la literatura especializada. Doleys define la encopresis funcional como la emisión de material fecal de cierta cantidad y consistencia en la ropa o en otros lugares inaceptables, en ausencia de alguna patología orgánica y a partir de los 3 años de edad. Por su parte, Levine considera que un niño padece encopresis cuando tiene más de 4 años y mancha su

Correspondencia: Andrés López de la Llave. Facultad de Psicología, Universidad Nacional de Educación a Distancia, Apartado 60148, 28040 Madrid. ropa interior regularmente con deposiciones formadas, semiformadas o líquidas, sin una etiología orgánica primaria. Ambos autores coinciden con la mayoría al excluir la presencia de organicidad como causa principal del problema, pero discrepan respecto al limite de edad requerido para asignar el diagnóstico de encopresis. Levine introduce una pauta de regularidad, sugiriendo que los accidentes ocasionales no deberían ser considerados; pero ninguno de los dos se pronuncia sobre otro de los aspectos que suscita polémicas entre los distintos autores, el carácter involuntario o voluntario, si bien parece aceptarse por la mayoría el carácter involuntario del problema. 
El DSM-IV define la encopresis como un trastorno de eliminación, cuya característica esencial consiste en la evacuación repetida de heces en lugares inadecuados (por ejemplo, ropa o suelo) cuando el niño ha superado los 4 años de edad o un nivel de desarrollo equivalente. Citando como criterio de exclusión, que la incontinencia no debe ser únicamente consecuencia del efecto ocasionado por la ingestión de ciertas sustancias (laxantes) o por la presencia de una enfermedad médica. El problema debe suceder como mínimo una vez al mes durante un periodo continuado de 3 meses. Por otro lado, el CIE-10 establece un criterio de duración de seis meses para considerar el diagnóstico de encopresis.

Como sucede con la mayoría de los trastornos infantiles, resulta difícil identificar con nitidez una etiología única de la encopresis, ya que la intervención concurrente de varios factores conduce necesariamente a una explicación multicausal del problema. Entre estos factores, el estreñimiento, un entrenamiento inapropiado y las experiencias desagradables asociadas a la defecación se perfilan como los elementos causales (Bragado, 1998).

Argumentos de corte fisiológico y conductual se entremezclan para explicar cómo contribuyen tales factores al desarrollo y mantenimiento del problema, reforzando la noción de que la encopresis es un trastorno psicofisiológico.

Sin embargo quedan por aclarar las diferencias sexuales encontradas en la distribución del trastorno, se desconoce por qué los varones son más propensos a padecerlo que las mujeres. Una posible razón, utilizada para explicar este mismo fenómeno en la enuresis (Bragado, 1994), podría ser que los varones siguen un curso de desarrollo más lento que las mujeres durante la primera infancia y la adolescencia. El hecho de que la encopresis descienda drásticamente con la edad y la casi inexistencia del trastorno a partir de los 15 años, apoyarían esta idea.

Las consecuencias sociales que siguen al ensuciamiento son bien conocidas y en muchos de los casos guardan una relación funcional con su disminución o mantenimiento. Estas consecuencias tienen gran importancia cuando el sujeto tiene edad escolar y los ensuciamientos se producen en este contexto, donde la coexistencia de la encopresis con otros problemas de conducta es algo habitual. Las conductas de oposición, el déficit de atención, encopréticos desobedientes e intrépidos y con fuertes cambios de humor o por el contrario con signos de ansiedad o tristeza, niños que tienden a evitar el contacto con sus compañeros y no participan en los juegos ni en las actividades deportivas, son algunos de los problemas que podemos encontrar.

El tratamiento conductual de la encopresis pone mucho empeño en identificar las conductas objeto de modificación, empleando estrategias que incluyen el uso de técnicas de reforzamiento positivo, castigo, reforzamiento negativo, control de estímulos y biofeedback. Algunos estudios han informado de buenos resultados utilizando sólo alguna de las técnicas citadas, por ejemplo el método de Neale (1963) sienta las pautas del entrenamiento en hábitos defecatorios, utilizando principalmente el reforzamiento social y material cuando el niño defeca en el inodoro; en contraste con este trabajo, Pedrini y Pedrini (1971) emplearon reforzamiento por permanecer limpio, comprobándose en ambos trabajos resultados satisfactorios. Sin embargo, la práctica habitual consiste en utilizar paquetes de tratamiento multicomponentes, empleándose varias de las técnicas citadas anteriormente.

En esta intervención se ha utilizado el reforzamiento positivo dirigido a incrementar tres tipos de conducta: sentarse 
en el water en momentos predefinidos, defecar en el inodoro y permanecer limpio. La utilización de técnicas de castigo, no gozan en general de la misma aceptación que las de reforzamiento positivo, no obstante, y siguiendo las recomendaciones de la mayoría de los expertos, se han utilizado la «sobrecorrección restitutiva» y la "practica positiva» como parte del programa pero sin otorgarles un papel preponderante.

\section{DESCRIPCIÓN DEL CASO Y PLANTEAMIENTO DE LA INTERVENCIÓN}

M. tiene siete años y cursa $2^{\mathrm{a}}$ año de primaria, es la menor de cuatro hermanos y desde su ingreso en el centro escolar (a los tres años y nueve meses) viene presentando episodios de ensuciamiento. Durante el primer año de su escolarización, conocida su encopresis, fue atendida por la Unidad de Salud Mental Infanto Juvenil (USMIJ). Por la entrevista mantenida con la madre se conoció que el inicio de la encopresis puede situarse cuando M. tenía tres años y cuatro meses al ser hospitalizada durante diez días tras el diagnóstico de encopresis pertinaz realizado por el Servicio de Urgencias.

El equipo de la USMIJ inició el tratamiento de la niña centrándose prioritariamente en el ámbito familiar. Parece que las recomendaciones relativas a la alimentación, al entrenamiento en hábitos de defecación, al uso del retrete, etc., no fueron llevadas a cabo con la suficiente frecuencia, lo que posiblemente condujo al mantenimiento de la encopresis.

La situación empeoró al iniciar la niña el primer curso de Educación Primaria a los cinco años y nueve meses; en ese momento, tras el fracaso de la intervención de la USMIJ, los problemas no sólo se circunscribían a los episodios encopréticos sino que se ampliaron a las rela- ciones conflictivas con sus compañeros. La pauta seguida por su tutora consistió en separar a la niña del resto de sus compañeros después de los sucesos de ensuciamiento, lo que conllevaba la escasa participación en trabajos de grupo y el aumento de conductas agresivas.

Antes de iniciar el tratamiento, la tutora (y terapeuta) citó a los padres de la niña mediante una carta en la que les informaba de la intención de llevar a cabo una intervención para tratar de solucionar el problema de su hija, y para pedirles su colaboración. Pasadas dos semanas de la citación y tras la no comparecencia de los padres, se solicitó la colaboración de la trabajadora social del barrio para que, a través de ella, se consiguiese la participación de los padres, intento que resultó también infructuoso. Dadas estas circunstancias, el Equipo de Ciclo determinó realizar la intervención exclusivamente en el contexto escolar.

Con los datos citados se elaboró un programa de tratamiento individualizado (Bragado, 1998), teniendo en cuenta el contexto escolar, y se programaron los objetivos específicos de la intervención sin olvidar la influencia de otras variables que afectarían al tratamiento, como:

- Partir del diagnóstico médico para constatar la ausencia de causas orgánicas.

- Informar a la niña y a sus compañeros de lo que se iba a hacer procurando que lo entendiesen y aceptasen.

- Potenciar las técnicas de reforzamiento positivo y minimizar el uso del castigo.

- Supervisar estrechamente la marcha del tratamiento para solucionar los problemas que puedan surgir en su puesta en práctica.

La intervención se desarrolló durante seis meses, siempre durante la jornada 
escolar que tenía un horario de nueve de la mañana a dos de la tarde, interrumpiéndose en los días festivos y de vacaciones. La persona que actuó como terapeuta fue la tutora de la clase.

\section{TRATAMIENTO}

\section{Evaluación inicial}

La evaluación conductual de la encopresis centra su foco de atención en averiguar si se han aprendido buenos hábitos de defecación e higiene $y$ en determinar las condiciones sociales, ambientales y personales que puedan estar interfiriendo con su aprendizaje o con su mantenimiento; es imprescindible obtener una información detallada sobre estos aspectos, dado que se necesita, además de comprender el problema, conocer todos los factores antecedentes y consecuentes que permitan establecer relaciones funcionales, terapéuticamente manejables (Bragado, 1998) (Tabla 1).

Los datos obtenidos de la primera entrevista con la madre aportaron el conocimiento del inicio del problema a los 3 años y 4 meses, momento en el que se hospitalizó a la niña con el diagnóstico de encopresis pertinaz. Antes de este suceso parece que la pauta defecatoria de M. era totalmente normal, no manifestando estreñimiento ni dolor al defecar y no abusaba de chocolates y colas, por lo que el diagnóstico que pareció más acertado fue el de encopresis secundaria.
Respecto a la frecuencia de ensuciamientos y el período horario de ocurrencia, la información se obtuvo de las entrevistas mantenidas con las profesoras de Educación Infantil y del registro de observación realizado por la tutora de $1^{9}$ durante cuatro meses, completándose el registro para establecer la línea base con otros dos meses de observación realizados por la terapeuta. El periodo horario de ocurrencia de los ensuciamientos fue de 9,30 a 11 de la mañana y la frecuencia aparece en la Tabla 6 (Línea Base).

La información recogida por la psicóloga del Centro y por las profesoras mostraron que las costumbres matinales observadas en su casa no permitían a la niña mantener pautas rutinarias de evacuación. Por otra parte, M. carecía de autonomía en su aseo, realizándoselo su madre, parece que no había adquirido hábitos elementales de limpieza, no sabe lavarse las manos, usa en pocas ocasiones los servicios del colegio y sus trabajos no son limpios.

\section{Intervención}

El análisis de la información de la que disponíamos centraba nuestra intervención en lograr que la niña instaurara en su repertorio la conducta de defecar en el lugar adecuado, los servicios del colegio; como sabemos la adquisición del control intestinal es una tarea compleja que depende del desarrollo neuromuscular y del aprendizaje de hábitos defecatorios.

Tabla 1. Objetivos y estrategias de la evaluación inicial

Objetivos

1. Identificar el tipo de encopresis

2. Delimitar la conducta problema: frecuencia de ensuciamientos y periodo horario de ocurrencia.

3. Determinar hábitos higiénicos: evitación total o parcial del uso del retrete, rutinas matinales antes de entrar en el colegio y autonomía en el aseo personal.
Estrategias

1. Entrevista con los padres.

2. Observación y registro de la frecuencia y momentos de los ensuciamientos.

3. Entrevistas con los profesores.

4. Entrevistas y recogida de información a los padres, profesores y compañeros. 
En los niños sin defectos anatómicos, y es este nuestro caso, el dominio de la continencia requiere fundamentalmente que aprendan a discriminar la sensación de distensión rectal y a responder a ella contrayendo voluntariamente el esfínter externo hasta llegar al lugar apropiado para defecar (Tabla 2).

Para consolidar el repertorio conductual del uso adecuado del baño, la técnica empleada ha sido la practica positiva. Se ensaya la conducta de ir hasta el servicio, sentarse en el WC contar hasta ochenta y volver de nuevo a la clase aunque no se haya defecado. Parece obvio que si un niño se sienta en el water un número de veces determinado, coincidiendo con los momentos más apropiados, la probabilidad de que se ensucie encima disminuye. La práctica positiva se realizó diariamente a las 10:30 h. ya que según los datos recogidos en la evaluación inicial, este era el momento más apropiado.

Durante las dos primeras semanas y con el objetivo de mantener relajada a la niña, acompañaron a $M$. al servicio varias compañeras de la clase, podían permanecer en el servicio hasta cinco minutos y si alguna de ellas necesitaba usar el water podía hacerlo. Cuando volvían a clase se las reforzaba con alabanzas.

Para realizar el moldeado de las conductas de lavarse, se dispone de un bolso que contiene todo lo necesario para el aseo, M. recoge el bolso de la percha de la clase y acompañada por la tutora va al servicio, donde se realizan todas aquellas conductas que preparan la limpieza de la niña para posibles sucesos de ensuciamiento. Una vez terminada la secuencia se refuerza a $M$. por lo bien que lo hace $y$ lo limpia que se encuentra. Este entrenamiento se realiza todos los días a las 9'30 de la mañana durante un periodo de $\mathbf{1 0}$ días consecutivos y posteriormente se realiza como recordatorio una vez a la semana. En la Tabla 3 se presenta el contenido de esta actividad.

En la tercera semana, se comienzan los ejercicios para desarrollar sensaciones de discriminación de distensión rectal. El objetivo es que la niña comience a centrar su atención en las sensaciones internas, para aumentar la percepción de las claves asociadas con el deseo de evacuar, y controlar los reflejos responsables de la defecación.

Los alumnos (un grupo de cuatro), se tumban en el suelo y comienzan a mover las piernas (pedaleo), pasados unos segundos se les manda parar y todos en silencio colocarán las manos en su vientre para intentar notar si se mueve, se repite varias veces y siempre en silencio para notar los movimientos del cuerpo concentrándose en sus sensaciones internas. Posteriormente se acompaña a la niña al servicio instruyéndola para reali-

Tabla 2. Objetivos y estrategias de la intervención

\section{Objetivos}

1. Consolidar el repertorio conductual, respecto al uso del baño.

2. Instaurar y/o aumentar hábitos de aseo personal.

3. Desarrollar sensaciones de discriminación de distensión rectal.

4. Reducir la agresividad y aumentar las interacciones con sus compañeros.

5. Desarrollar interés y motivación para estar limpia.

\section{Estrategias}

1. Práctica positiva.

2. Moldeado y entrenamiento en hábitos de aseo personal.

3. Ejercicios para aumentar la percepción del deseo, y controlar los reflejos responsables de la evacuación.

4. Extinción de las conductas inadecuadas, y refuerzo social de las adecuadas.

5. Técnica de economía de fichas, con un procedimiento de contingencia de grupo.

6. Sobrecorrección, inmediatamente después de los ensuciamientos. 
Tabla 3. Descripción del entrenamiento que realiza $M$. todos los días

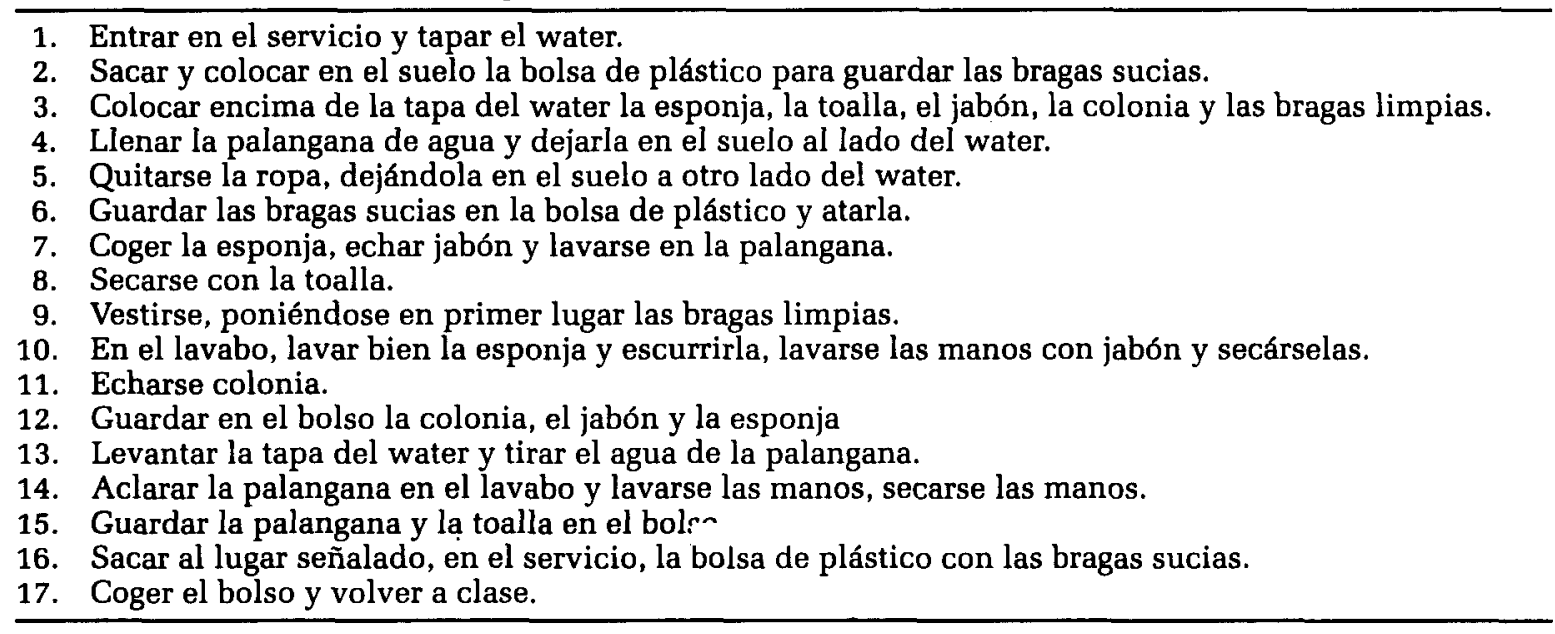

zar ejercicios esfintéricos de contracciónrelajación. Se le dice que «apriete el esfínter» como si tratara de impedir el escape de gases, empleando una analogía con la interrupción del chorro de orina, o que empujara como si fuera a defecar. Posteriormente debe quedarse sentada en el water repitiendo varias veces los ejercicios, y volver a clase. A partir de esta sesión la practica positiva se realiza al terminar los ejercicios.

Con el objeto de aumentar las interacciones positivas con sus compañeros, durante la primera semana de intervención, se realizó un registro de aquellos niños que mostraban mas interés por ser amigos de M. Partiendo de este registro, en la segunda semana se reorganizó la clase agrupando a los alumnos en mesas de cuatro, colocando a $M$. con dos niñas y un niño, instruyéndoles para que cada vez que ésta emitiera alguna de las conductas inadecuadas seleccionadas previamente (agredir, arrojar objetos, levantarse de la mesa, interrumpir y estropear el trabajo y apropiarse del material), no pidieran ayuda a la tutora, sino que en silencio se acercaran a su mesa y señalaran con una cruz la conducta emitida en la hoja de registro 2 (ver apéndice). Simultáneamente se realizó un programa de reforzamiento, utilizando las alaban- zas, la sonrisa y el elogio como reforzadores, cada vez que ocurría alguna de las conductas adecuadas: permanencia correcta en su sitio, dirigirse con agrado a los compañeros y pedir prestado el material.

Como la intervención tenia como objetivo la integración de la niña con el resto de sus compañeros de clase, se mantuvo este grupo durante dos semanas, alternando posteriormente a los componentes del grupo, de tal forma que la niña pudiera interaccionar con todos los alumnos.

El reforzamiento positivo es sin duda el método más extendido para abordar el problema de la encopresis. Con objeto de promover hábitos de defecación apropiados, el reforzamiento positivo se ha dirigido fundamentalmente a incrementar tres tipos de conducta: sentarse en el inodoro en momentos predefinidos, defecar en el WC y permanecer limpio. Aunque no se ha investigado específicamente qué tipo de reforzamiento es más eficaz para lograr el éxito, existen serias dudas respecto a la conveniencia de reforzar la conducta de permanecer limpio, sobre todo en lo que respecta a la encopresis retentiva. Muchos autores ( Doleys, McWhorter, Williams y Gentry, 1977; Steege y Harper, 1989; etc.) adoptan la postura de combinar el reforzamiento por 
defecar en el water con el de mantenerse limpio.

Dado nuestro especifico contexto, y teniendo en cuenta el corto periodo temporal del que se disponía y aún conociendo todo lo anterior, decidimos utilizar el reforzamiento por mantenerse limpia como objetivo para desarrollar la motivación, teniendo en cuenta que el rechazo de sus compañeros era debido principalmente a su mal olor y que evitando éste conseguiríamos una mayor integración en el grupo.

Para aumentar la motivación se utilizo un programa basado en recompensas, con un procedimiento de contingencia de grupo y que se caracteriza porque un alumno ( $M$. ) gana recompensas contingentes a su comportamiento, pero que son compartidas por toda la clase. Se utilizó como sistema de reforzamiento, la economía de fichas, ganándose estas cada día que la niña terminaba la jornada escolar manteniéndose limpia.

La fórmula elegida, consistió en colocar en la pared de la clase un póster con la lista de los nombres de todos los alumnos, donde se escribió « M. gana soles para sus compañeros», y otro póster que decía "Los compañeros ganan soles para M.», y sólo estaba el nombre de la niña. Cada día a la salida, cuando $M$. se encontrase limpia, llamaría al compañero de la lista y este pegaría un sol en su póster, ella a su vez pegaría otro al lado del nombre del compañero en el otro póster. Cuando en el primer póster cada niño tuviera su sol, se celebraría una gran fiesta en el pinar cercano al Colegio, con juegos y chucherías. Se determinó también que no se pegarían soles en los pósters cuando no se cumpliesen las circunstancias señaladas.

Se comprobó que todos comprendieran correctamente el proceso y que conocieran el premio que la clase conseguiría con $\mathrm{M}$.

La sobrecorrección se utilizó inmediatamente después de los sucesos de ensu- ciamiento, su característica esencial consiste en que el niño repare el daño que ha ocasionado con su conducta, a fin de restablecer las condiciones ambientales preexistentes a la comisión del acto: ensuciarse. En estos casos, la niña se iba al servicio con la bolsa de aseo, realizando la secuencia de conductas de la Tabla 3.

\section{Evaluación de la intervención}

La técnica de evaluación utilizada ha sido la observación directa y el registro diario de las conductas que aparecen en el Cuadro 3. Los marcos de observación han sido el aula y los lavabos del colegio. Se han empleado dos tipos de hojas de registro (ver apéndice), en la primera, además del número de ensuciamientos y de salidas al lavabo, se han registrado las diferentes estrategias llevadas a cabo en la intervención, así como el horario de realización de las mismas, con el objetivo de mantener un seguimiento para realizar las modificaciones necesarias. En la segunda, los compañeros de mesa de $\mathrm{M}$. registraban todas aquellas conductas inadecuadas emitidas por ésta durante el desarrollo de la clase (Tabla 4).

En la Tabla 5, a modo de resumen, se presenta la frecuencia mensual de las conductas objetivo durante la intervención, y en Tabla 6, la frecuencia mensual de los episodios encopréticos durante la línea base, la intervención y el seguimiento.

\section{CONCLUSIONES Y DISCUSIÓN}

Parece que la aplicación del programa ha resultado eficaz puesto que los episodios de encopresis se redujeron a 1 entre las semanas 15-19 de tratamiento, y posteriormente, entre la semana 20 y 24 , quedaron reducidos a cero, dándose por concluido en el momento en que se había 
Tabla 4. Conductas objetivo de la evaluación del tratamiento

\begin{tabular}{|c|c|}
\hline Conductas objetivo & Definición \\
\hline 1. Episodios encopréticos. & $\begin{array}{l}\text { - Deposiciones en la ropa interior en lugares ina- } \\
\text { decuados (aula y patio de recreo). }\end{array}$ \\
\hline 2. Salidas al lavabo. & $\begin{array}{l}\text { - Solicitar permiso a la tutora para salir del aula } \\
\text { e ir al lavabo. }\end{array}$ \\
\hline 3. Agresión a los compañeros de mesa. & $\begin{array}{l}\text { - Pellizcar, tirar del cabello, abofetear, insultar y } \\
\text { dar patadas a sus compañeros durante el desa- } \\
\text { rrollo de las clases. }\end{array}$ \\
\hline 4. Tirar objetos al suelo & $\begin{array}{l}\text { - Coger material de clase (lápices, gomas, regla...) } \\
\text { y arrojarlos al suelo. }\end{array}$ \\
\hline 5. Levantarse de su sitio sin permiso & $\begin{array}{l}\text { - Levantarse de su mesa para acercarse a otras } \\
\text { mesas, al perchero y a las ventanas. }\end{array}$ \\
\hline $\begin{array}{l}\text { 6. Utilizar material de sus compañeros sin per- } \\
\text { miso }\end{array}$ & $\begin{array}{l}\text { - Emplear la goma, lápices de colores, etc. de sus } \\
\text { compañeros para uso propio. }\end{array}$ \\
\hline $\begin{array}{l}\text { 7. Interrumpir y estropear el trabajo de sus com- } \\
\text { pañeros }\end{array}$ & $\begin{array}{l}\text { Echarse sobre la mesa encima del cuaderno de } \\
\text { sus compañeros impidiéndoles su trabajo y } \\
\text { arrugar, romper y pintar en los cuadernos de } \\
\text { los demás. }\end{array}$ \\
\hline
\end{tabular}

Tabla 5. Frecuencia mensual de las conductas objetivo durante la intervención

\begin{tabular}{ccccccc}
\hline Mes & Salidas al lavabo & $\begin{array}{c}\text { Agresión a } \\
\text { compañeros }\end{array}$ & $\begin{array}{c}\text { Levantarse } \\
\text { sin permiso }\end{array}$ & Tirar objetos & $\begin{array}{c}\text { Usar material } \\
\text { sin permiso }\end{array}$ & $\begin{array}{c}\text { Interrumpir y } \\
\text { estropear trabajo }\end{array}$ \\
\hline 1 & 9 & 38 & 29 & 32 & 15 & 22 \\
2 & 12 & 25 & 18 & 23 & 11 & 18 \\
3 & 8 & 8 & 7 & 18 & 9 & 16 \\
4 & 20 & 15 & 8 & 16 & 6 & 7 \\
5 & 19 & 15 & 7 & 9 & 3 & 7 \\
6 & 25 & 10 & 3 & 10 & 1 & 7 \\
\hline
\end{tabular}

Tabla 6. Frecuencia mensual de los episodios encopréticos

\begin{tabular}{cccccc}
\hline Mes & Línea Base & Mes & Intervención & Mes & Seguimiento \\
\hline 1 & 11 & 7 & 5 & 13 & 1 \\
2 & 10 & 8 & 3 & 14 & 1 \\
3 & 9 & 9 & 0 & 15 & 0 \\
4 & 9 & 10 & 1 & 16 & 0 \\
5 & 10 & 11 & 1 & 17 & 0 \\
6 & 8 & 12 & 0 & 18 & 0 \\
\hline
\end{tabular}

logrado el criterio de éxito (4 semanas sin encopresis).

Se realizó un seguimiento durante los seis meses siguientes para observar la ocurrencia de los sucesos de ensuciamiento, apareciendo uno al mes durante los dos primeros meses, reduciéndose a cero en los cuatro meses siguientes (ver Tabla 3 y Figura 1). Respecto al resto de conductas objetivo, se ha observado, durante los seis meses de seguimiento, que han descendido notablemente, con una frecuencia mensual inferior a 1, las de levantarse sin permiso, tirar objetos y usar material sin permiso. Las conductas de agresión a compañeros e interrumpir y estropear trabajos, se sitúan en una frecuencia mensual de 2,5. Por otra parte, las salidas al lavabo siguen manteniendo una frecuencia alta (20 salidas mensuales). 
En general, la niña usaba el baño correctamente, si bien dadas las circunstancias de poder salir de la clase cuando ella tuviera necesidad, la frecuencia de salidas era muy superior a la del resto de sus compañeros (Figura 2), explicando siempre éstas por motivos que se consideraban correctos: lavarse las manos, hacer pis, hacer caca, beber agua., este elevado número de salidas obstaculizaba enormemente su rendimiento escolar, lo que nos hace pensar que debería programarse de forma diferente la intervención para el uso correcto del servicio, incidiendo en la importancia de un buen uso.
Respecto a los resultados en el entrenamiento de conductas de limpieza, puede decirse que estos son excepcionales, no sólo se ha conseguido que las lleve a cabo en los momentos que se ha ensuciado, haciéndolo correctamente, sino que además ha mejorado notablemente su aspecto de aseo personal, acude a clase más limpia, huele a colonia y mejor peinada.

Así mismo, ha supuesto un notable cambio, respecto a la situación inicial, la emisión de las otras conductas desadaptativas que fueron objeto de intervención; así, se redujo notablemente la apropiación del material de los compañeros y la
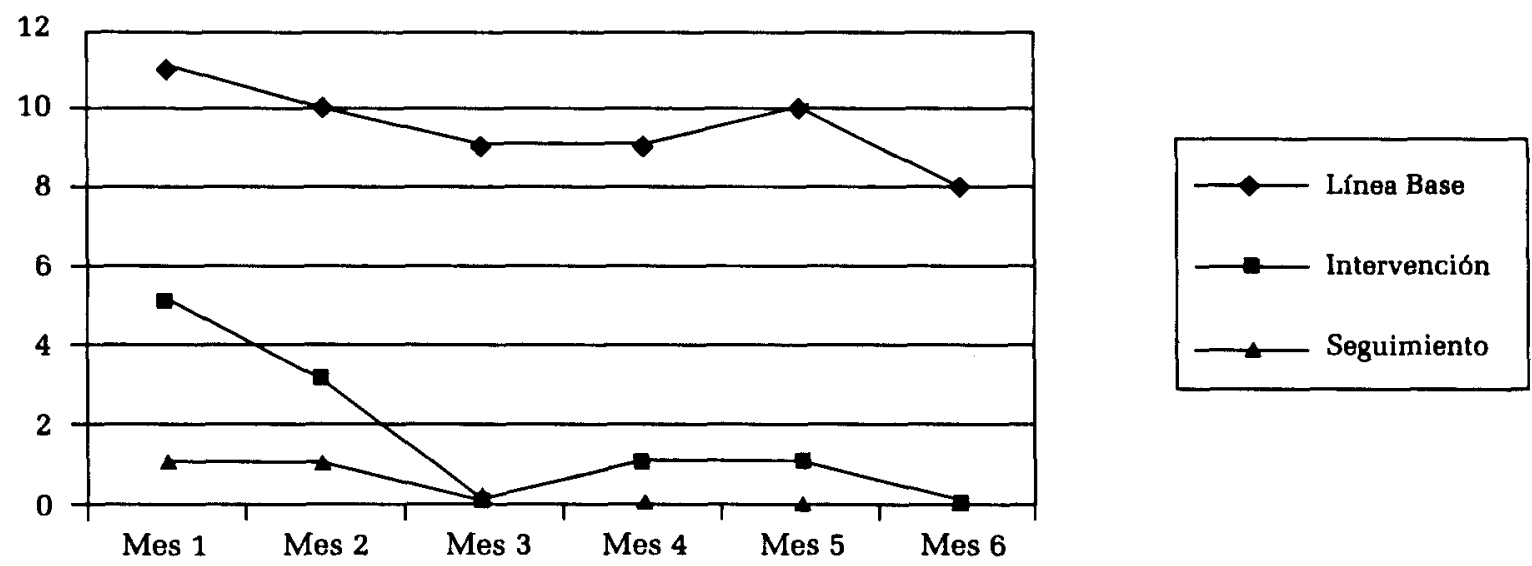

Figura 1. Frecuencia mensual de episodios encopréticos

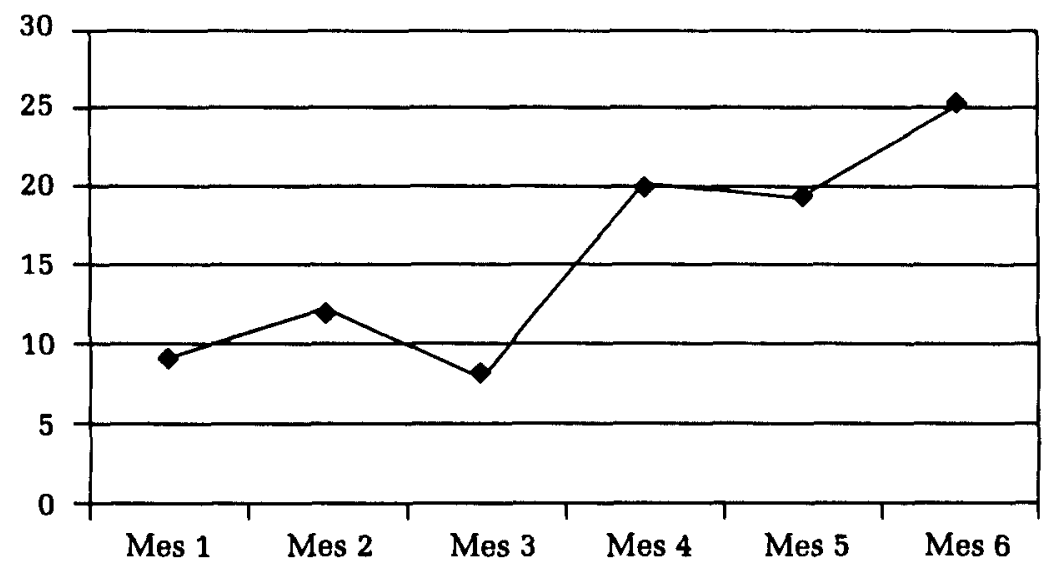

Figura 2. Frecuencia mensual de salidas al lavabo 
conducta de levantarse de la mesa (Figuras 3 y 4). Este resultado podría deberse al programa de reforzamiento social llevado a cabo sobre las conductas incompatibles, pedir prestado el material y permanecer sentada.

El notable aumento en el número de interacciones positivas con los compañeros, ha sido debida probablemente, al descenso y desaparición de los sucesos de ensuciamiento lo que ha favorecido la integración en juegos y actividades grupales, y la no manifestación de agresividad; si bien la aplicación de recompensas, en mayor medida las alabanzas y elogios, ha supuesto una enorme interferencia en la consecución de los objetivos específicamente educativos, circunstancia esencial en el contexto en el que nos encontramos.

Esta interferencia se manifiesta en la no aceptación de las criticas por su bajo rendimiento, que la dirige su maestra cuando presenta las tareas inacabadas, nótese que ha sido la persona que ha realizado la intervención y que como terapeuta ha proporcionado refuerzo social y recompensas contingentes a sus conductas adecuadas para la desaparición de la encopresis; lo que nos puede llevar a

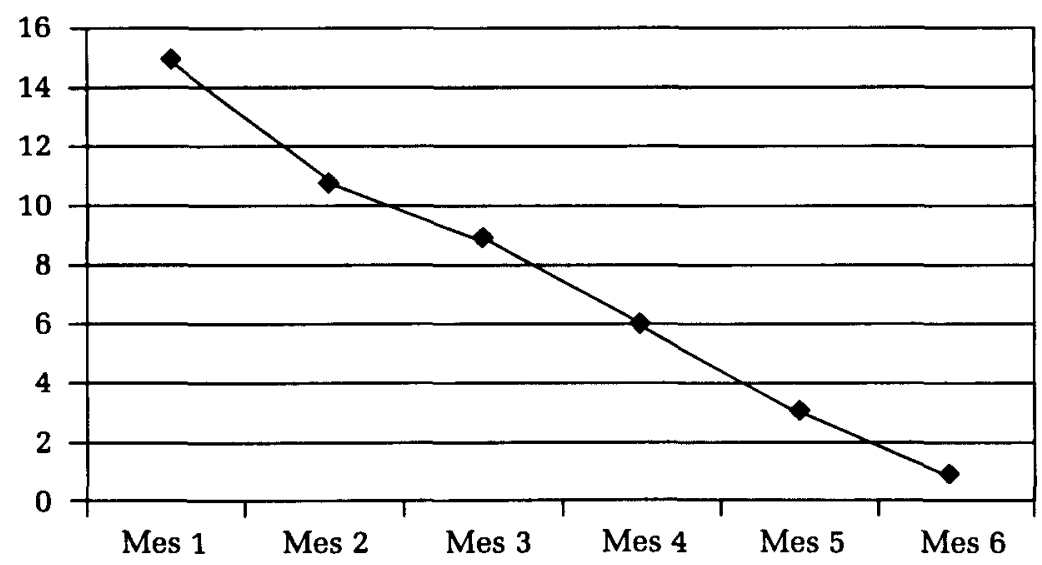

Figura 3. Frecuencia mensual de apropiaciones de material sin pedir permiso
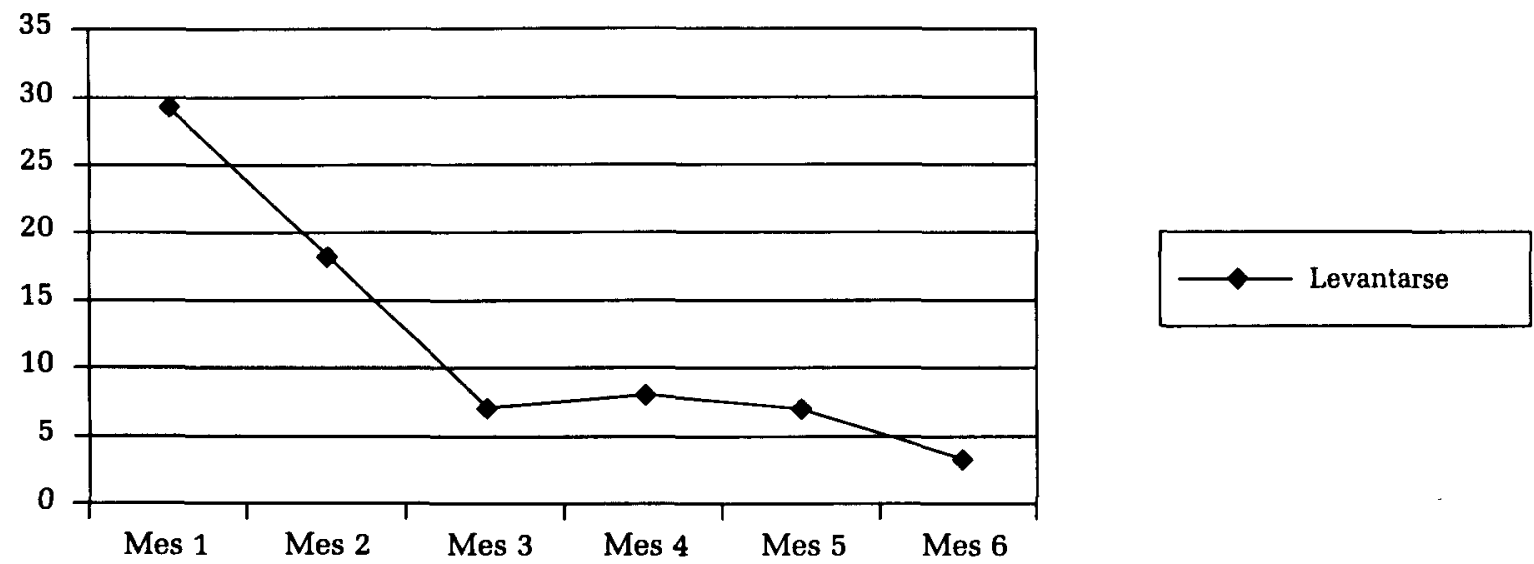

Figura 4. Frecuencia mensual de la conducta de levantarse de su sitio sin permiso 
hipotetizar que M. la acepta en su rol de terapeuta y que no entiende que es a la vez su tutora y por tanto debe actuar como tal. Todo lo anterior nos lleva a la conclusión del especial cuidado que debe ponerse en la persona que actuará como terapeuta, ya que sin duda su intervención es esencial y no debe interferir con otras, como en el caso que describimos, donde la consecución de los objetivos educativos es prioritario. Sería interesante tomar esta consideración en cuenta en otras posibles intervenciones en el contexto escolar.

En resumen, puede afirmarse que $M$. ha conseguido instaurar hábitos defecatorios adecuados y ha eliminado los ensuciamientos, consiguiendo integrarse en el grupo de clase manifestando menor agresividad. Esta evolución favorable puede atribuirse, muy probablemente, como se ha visto a lo largo de la anterior discusión, a la intervención terapéutica realizada.

\section{REFERENCIAS BIBLIOGRÁFICAS}

American Psychiatric Association (1994). Diagnostic and Statistical Manual of Mental Disorder ( $4^{a}$ ed.). Washington (Edición española, Barcelona: Masson, 1995).

Barlow, D. y Hersen, M. (1984). Diseños experimentales de caso único. Barcelona: Martínez Roca.

Bragado, C. (1998). Encopresis. Madrid: Pirámide.

Caballo, V.E. (1993). Manual de Técnicas de Terapia y Modificación de Conducta. Madrid: Siglo XXI de España Editores.

Carrobles, J.A. (1996). Análisis y Modificación de Conducta II. Madrid: UNED.

Kazdin, A.E. (1978). Modificación de Conducta y sus aplicaciones Prácticas. México: El Manual Moderno.

Leitenberg, H. (1976). Modificación y Terapia de Conducta. Madrid: Morata.

Vallejo, M.A. y Ruiz, M.A. (1993). Manual práctico de modificación de conducta. Madrid: Fundación Universidad Empresa. 


\section{Apéndice}

Hoja de registro 1

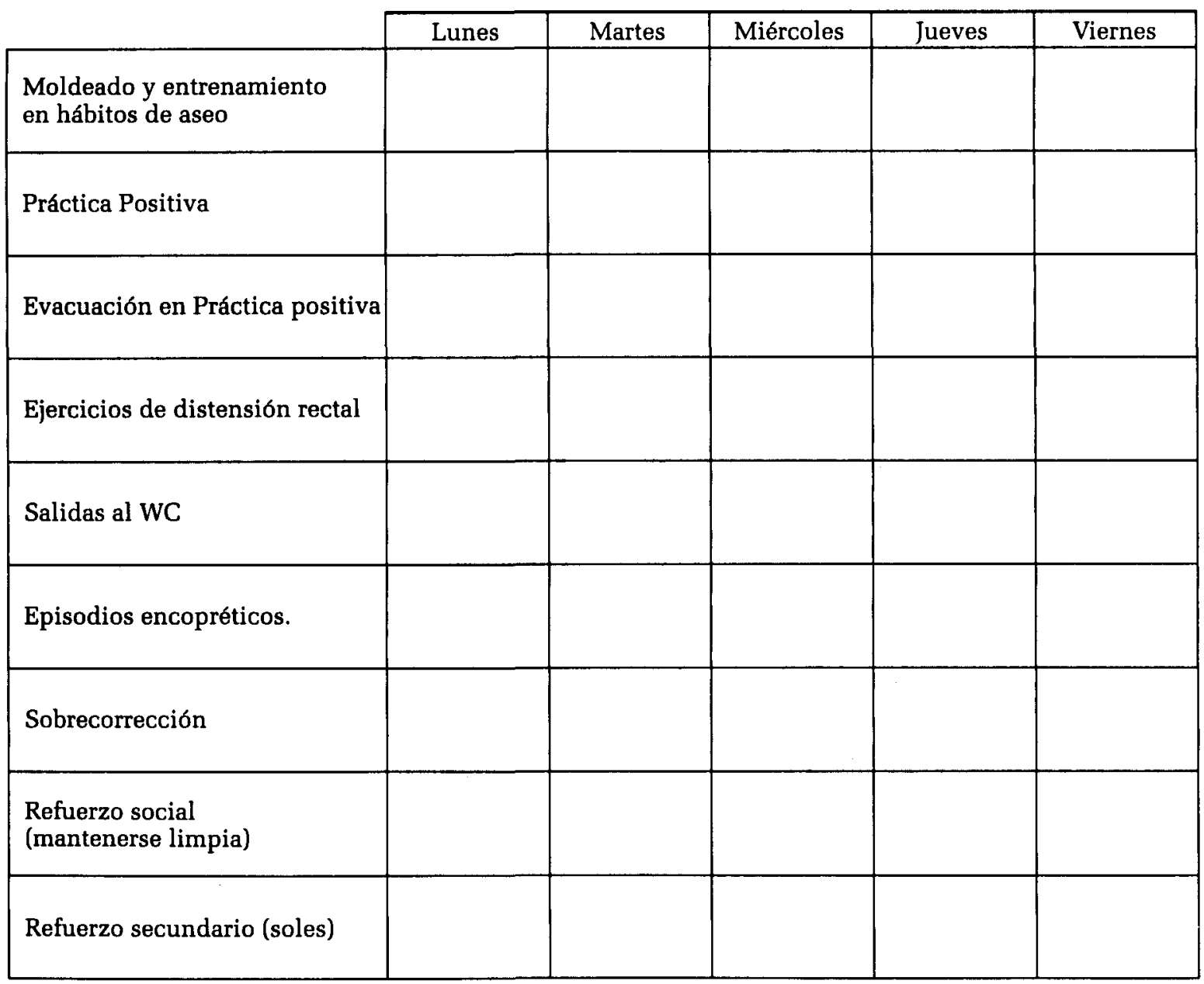

Hoja de registro 2

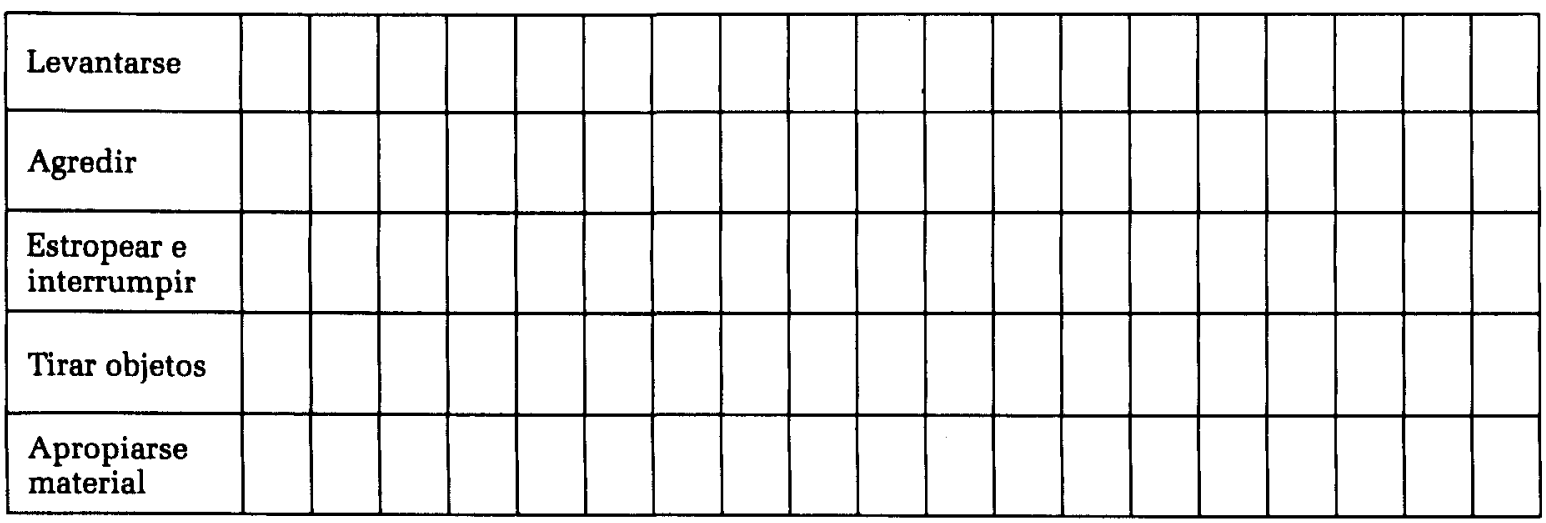

